\title{
Alteration of Helium-Filled Bubbles and Space Weathered Material During Heating in the TEM
}

\author{
K. D. Burgess ${ }^{1}$ and R. M. Stroud ${ }^{1}$ \\ 1. U.S. Naval Research Laboratory, Washington, DC.
}

Vesicles present in space weathered rims of some lunar soil grains are inferred to be due to the build-up of solar wind $\mathrm{H}$ and He. Water (i.e., hydrogen) has been tentatively identified in vesicles in space weathered rims of an IDP pyroxene grain [1]. However, helium has not been identified in situ in naturally irradiated materials. In the current study, we used spatially resolved electron energy loss spectroscopy (EELS) in a scanning transmission electron microscope (STEM) to demonstrate the presence of helium in vesicles in the space weathered rim of a lunar ilmenite grain and link the bubble characteristics to other previously observed space weathering features.

The samples were prepared using focused ion beam (FIB) microscopy. Regions of an ilmenite grain from lunar soil 71501 were coated with a thick carbon film $(1-2 \mu \mathrm{m})$ before ion milling to prevent damage to the grain surface by the ion beam. The thinned sections were moved directly from the FIB to the pre-bake vacuum chamber to drive off adsorbed water before insertion in the UHV system. EELS and energy dispersive X-ray spectroscopy (EDS) data were collected with the NION UltraSTEM200-X at the U.S. Naval Research Laboratory, equipped with a Gatan Enfinium ER EEL spectrometer with and a Bruker SDD-EDS detector. The STEM was operated with at $200 \mathrm{kV}$, with a 0.1 to $0.2 \mathrm{~nm}$ probe. Spectra were collected as spectrum images (SI), with a spectrum collected for each pixel, allowing for mapping of variations in thickness and composition.

The FIB sections expose multiple original grain surfaces in different orientations, and significant differences are seen in defect areal density and vesicle number in different regions. Solar flare tracks are not observed, but 71501 has a bulk cosmic ray exposure age $100 \mathrm{Myr}$ [2]. However, individual soil grains can be highly variable, and helium saturation and bubble formation could occur in $<500 \mathrm{yr}$ [3]. The general morphology closely resembles that observed previously in lunar and experimentally irradiated ilmenite $[4,5]$, with the space weathered rim consisting of a disordered outer layer rich in nanophase metallic iron $\left(n p F e^{0}\right)$ inclusions on most of the grain, covering an inner, defect-rich layer (Fig.1). The defects in the inner layer are aligned on the (001) plane of the ilmenite, regardless of the orientation of the exposed face. The outer layer of the ilmenite also contains a number of vesicles or bubbles. The concentration of helium in some bubbles ranges from $20-130 \mathrm{He} / \mathrm{nm}^{3}$ based on the method of Walsh et al. [6], and peak energies range from $22.0 \mathrm{eV}$ to $24.3 \mathrm{eV}$. Helium concentration in bubbles can vary over very short length scales.

Following the initial characterization, the samples will be heated using a Protochips Aduro sample holder (Fig. 2) in a JEOL 2200FS, and changes in bubble shapes and sizes, as well as other changes in sample morphology will be observed. We will heat the sample to temperatures comparable to atmospheric heating of IDPs [7] or those used in laboratory extraction experiments [8]. There are clear spatial and orientation relationships in the ilmenite between $\mathrm{nFFe}^{0}$ and some bubbles, indicating that growth of either inclusions or bubbles during annealing could affect the size and shape of surrounding features. Nanophase $\mathrm{Fe}^{0}$ is known to grow rapidly at high temperatures [9], while the internal pressure of the bubbles will increase with the expansion of helium upon heating. Helium could be lost from some bubbles through diffusion prior to the pressure exceeding the limits of the ilmenite structure as $n p \mathrm{Fe}^{0}$ growth creates cracks in the surface, but it is also possible that some bubbles will "pop" in a catastrophic failure, releasing a relatively 
large volume of helium very quickly. This effect is likely closely tied to the crystal orientation, as was noted for the measured helium density at room temperature.

Natural lunar and asteroidal samples experience daily temperature cycling (e.g., >300 K between lunar day and night), as well as extreme heating during impact events. Understanding how the complex nanostructures associated with space weathering are affected by heating, particularly the growth or rupture of helium bubbles, will provide insight into exospheric cycling of gases on the Moon and Mercury and transport and release of solar wind helium and other noble gases throughout the Solar System. Additionally, in situ observation of the alteration will contribute to a fuller understanding of how npFe ${ }^{0}$, vesicles, crystal structure, and temperature interact to influence the final space weathered characteristics [10].

References:

[1] Bradley, J.P., et al. PNAS, 111 (2014) p. 1732.

[2] Signer, P., et al. LPSC 8 (1977) p. 3657.

[3] Nichols, R., et al. GCA 58 (1994) p. 1031.

[4] Christoffersen, R., et al. LPSC 41 (2010) p. 1532.

[5] Christoffersen, R., et al. M\&PS 31 (1996) p. 835.

[6] Walsh, C.A., et al. Philos Mag A 80 (2000) p. 1507.

[7] Flynn, G.J. Icarus 77 (1989) p. 287.

[8] Nier, A.O., and D.J. Schlutter Meteoritics 27 (1992) p. 166.

[9] Thompson, M.S., et al. M\&PS (2016)

[10] The authors acknowledge funding from the RIS ${ }^{4}$ E node of NASA's Solar System Exploration Research Virtual Institute.

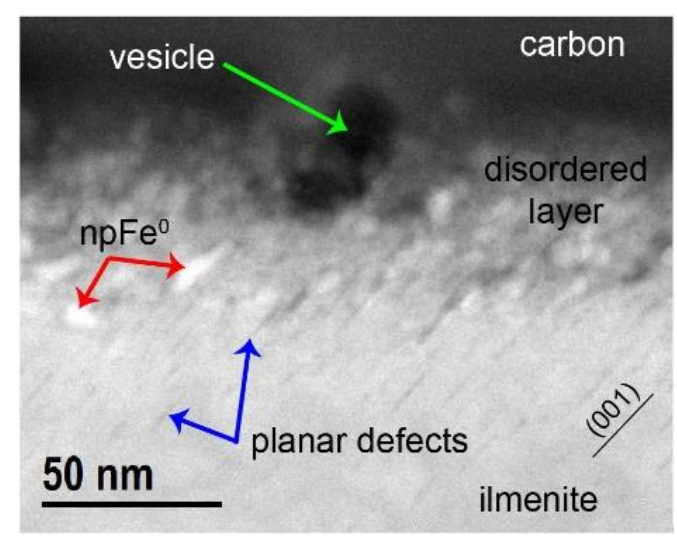

Figure 1. HAADF image of the space weathered rim of an ilmenite grain prior to heating, showing the presence of a large vesicle in a disordered, $\mathrm{nFe}^{0}$-rich rim, extending into a Si-rich vapor deposited rim. Planar defects are aligned on the (001) plane of ilmenite.

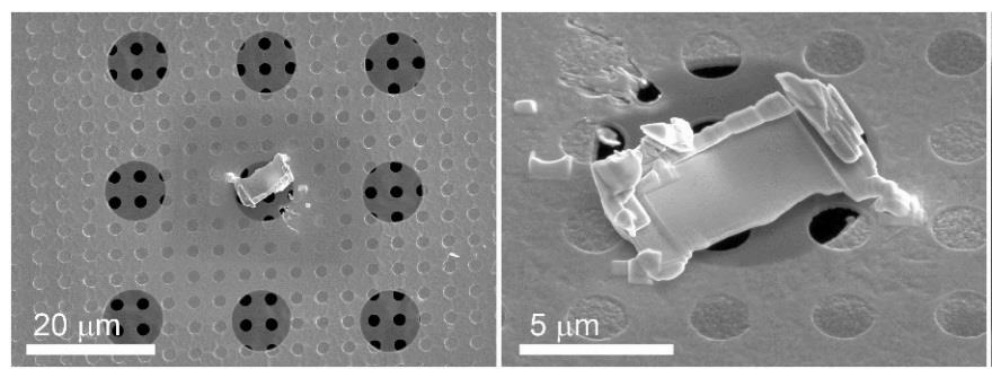

Figure 2. Secondary electron images of FIB sample on the chip used for heating. 